\title{
Applications of Timer of Data Sampling Software
}

\author{
Hongyan ZHUO*, Chengliang GE, Zhiqiang LIU, Jiaru ZHANG \\ Institute of Applied Electronics \\ CAEP, P.O. Box 919-1011 \\ Mianyang 621999, China \\ *E-mail: 526756092@qq.com \\ $+*$ Corresponding author
}

\begin{abstract}
Based on periodic inspection and scanning, data sampling software usually used WM_TIMER and MM_TIMER. MM_TIMER is a multimedia timer. There are differences between WM_TIMER and MM_TIMER, such as implementation methods, $P R I$ and precisions of time. Herein, the great differences are compared with experiments. Its precision will be reached at $1 \mathrm{~ms}$ with applications of MM_TIMER.
\end{abstract}

Keywords-periodic inspection and scanning; precision of timer; MM_TIMER; WM_TIMER

\section{INTRODUCTION}

Up to today, there are two methods of data sampling system based on periodic inspection and scanning. One method is using embedded system to achieved microsecond precision by hardware clock. Another method is using $\mathrm{A} / \mathrm{D}$ card to achieved millisecond precision. In the second method, most of users developed applied software based on Windows system. And for such multitask and multiuser system of Windows, developers cannot operate the rock-bottom hardware. Then, developers achieved periodic data sampling by STK of applied software [1]. MM_TIMER and WM_TIMER are usually used timers of VC by developers. And there are great differences of precision between two timers. So, the methods and precisions of the two timers are presented in this paper.

\section{DESIGN AND IMPLEMENTATION OF WM_TIMER}

WM_TIMER is a timer which is provided by Windows. The method is easy and simple. Developer can transfer SetTimer( ) function to allocate a timer to application program. While a time interval is specified, Windows system sends a WM_TIMER message every specified time interval. Then the program can implement events in real-time.

The procedure of implementation is listed as initialing of SetTimer( ), closing timer with KillTimer( ), sending message queue, sampling data in response function of WM_TIMER.

Function of SetTimer is defined as below.

UINT_PTR SetTimer(

UINI_PTR nIDEvent,

//identification code of timer

UINT nElapse,

//time interval with unit of $m s$

$\operatorname{Void}\left(C A L L B A C K^{*}\right.$

lpfnTimer)HWND,UINT,UINT_PTR,DWORD)

);

Wherein, parameter lpfnTimer is the callback function of timer message. If it is NULL, WM_TIMER sends message queue of program and will be disposed by OnTimer member fuctions. The return value 0 means fault.

For a data sampling system, codes of data sampling with CFormView basic class is listed below.

//setup timer under pushbutton of 'Data Sampling'

void CTestView::OnButtonRun()

\{ here

// TODO: Add your control notification handler code are $20 m s$

SetTimer(1,20,NULL); $\quad$ //sampling periods

\}

//implementation of data sampling

void CTestView::OnTimer(UINT nIDEvent)

\{

// TODO: Add your message handler code here and/or call default

writing functions of data sampling

,

// close timer under pushbutton of 'Stop Sampling'

void CTestView::OnButtonRun()

\{ here

// TODO: Add your control notification handler code KillTimer(1); \}

\section{DESIGN AND IMPLEMENTATION OF MM_TIMER}

MM_TIMER is a multimedia timer provided by Windows system. Within VC software, its difficult than WM_TIMER. The main procedure is to setup parameters of MM_TIMER, to transfer timeGetDevCaps( ) function to decide supported minimal precision and maximal precision, to startup timer to do applications, to release sources by deleting timer. The more used functions and means are listed below [2,3].

timeBeginPeriod( ) //setup minimal resolution of timer

timeEndPeriod( ) //eliminating minimal resolution of timer built by ascending function timeSetEvent( ) //producing time of allocated period

timeKillEvent( ) //deleting events of front timer

The interface functions of MM_TIMER are included in DLL of mmsystem.dll. And VC++ provides response head files. The detail implementation methods are listed below.

(1)Setup resolution of Timer 
MMRESULT timeGetDevCaps(lpTIMECAPS\&ptc, UINTcbtc) transfers timeGetDevCaps() function to judge the minimal resolution and maximal resolution. Then it transfers timeBeginPeriod(UINTuPeriod) function to setup resolution of timer. The parameter UINTuPeriod is the value of timer resolution.

(2)Setup events of timer

MMRESULTtimeSetEvent(UINT uDelay, //uDlelay: interval of sampling

UINT uResolution, // uResolution: precision of time, its default value is $1 \mathrm{~ms}$

LPTIMECALLBACK lpTimeProc, I/lpTimeProc: user defined callback //function

DWORD dwUser, UINT fuEvent); //dwUser: user provided callback function

// UINT fuEvent: mode of event, triggered by TIMER period

(3)Declaring of whole callback function

Void CALLBACK TimerCallBackProc(UINT wTimerID, UINT mMsg, DWORD dwUser, DWORD $d w 1$, DWORD $d w 2$ )

Wherein, wTimerID is sign of timer. $m M s g$ is reserved. $d w U s e r$ is parameter used by user. $d w 1$ and $d w 2$ are reserved.

(4)user defined message disposal function

The message disposal function defined by user is to receive messages from $M M_{-}$TIMER.

\section{APPLICATIONS}

\section{A. Experiments}

Above methods are applied in one big system. The curves of two timers are showed in figure 1 and figure 2 . Figure 1 is curves of sampled data with applications of WM_TIMER. The precision of WM_TIMER is $15 \mathrm{~ms}$. But, there is 'Losing Second' phenomenon. The maximal losing second is about $2 \mathrm{~s}-4 \mathrm{~s}$. As showed in figure 1 , the real sampling time is $18 \mathrm{~s}$ and figure 1 has only $16 \mathrm{~s}$. The $2 \mathrm{~s}$ is lost.

Figure 2 is curves of sampled data with applications of MM_TIMER. The precision of MM_TIMER is $1 \mathrm{~ms}$. And, there is no 'Losing Second' phenomenon. As showed in figure 2 , the real sampling time is $18 \mathrm{~s}$ and figure 2 has data with time duration of $18 \mathrm{~s}$. Time is not lost.

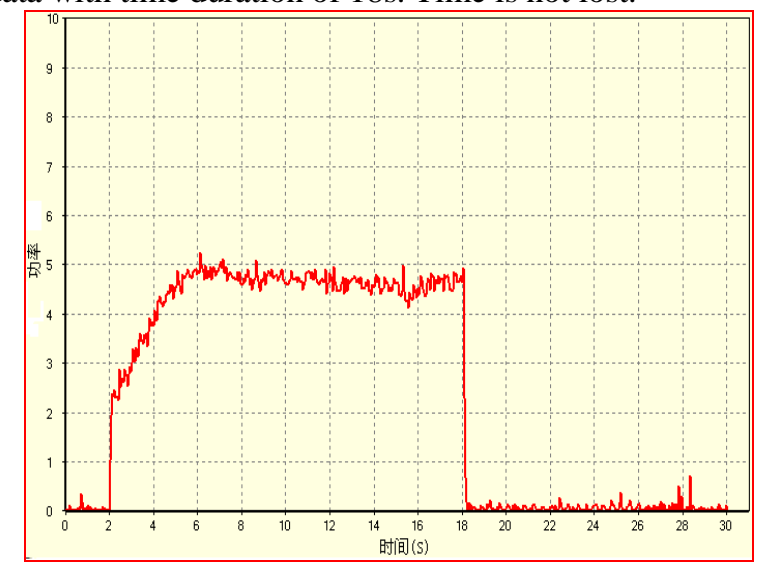

Figure 1. Data sampling curves of WM_TIMER

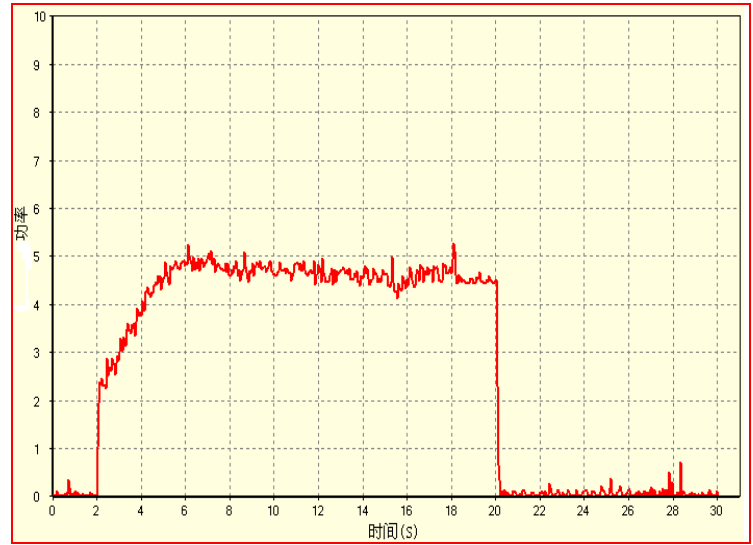

Figure 2. Data sampling curves of MM_TIMER

\section{B. Analysis}

After doing many experiments, it can be found that WM_TIMER is a simple extending of IBM PC hardware and timer logic built by ROM BIOS. ROM initializes the timer chip of Inter8254 to produce interrupt $08 \mathrm{H}$. Frequency of interrupt $08 \mathrm{H}$ is about $18.2 \mathrm{~Hz}$. BIOS updates two timer variables of TIMER-LO and TIMER-HI with interrupt $08 \mathrm{H}$. Hence, the maximal fault of WM_TIMER is that its resolution is 55ms [4]. That is, the program can receive 18 messages per second at best. And the PRI of WM_TIMER in Windows system is very low. While there are several WM_TIMER events, Windows system combines the events. Then there will be some events lost by Windows.

For mechanism of implementation of MM_TIMER, $M M \_T I M E R$ provides hardware interrupt. MM_TIMER has its independent thread to transfer its callback function. It has high PRI. Its precision can be achieved to $1 \mathrm{~ms}$ [5].

In the applications, it is watchful that it must be transfer MM_TIMER before end of the thread. Otherwise, the system will be done. And after application of MM_TIMER, the timer and its functions must be deleted. Otherwise, the system will be changed slowly and slowly.

\section{5 CONCLUSIONS}

By studying of applications, some conclusions are listed in table 1. From table 1, applications without requirements of high precision can apply WM_TIMER. And for an application with high sampling frequency and precision, the MM_TIMER is needed.

TABLE I. COMPARISON OF TWO TIMERS

\begin{tabular}{|c|c|c|}
\hline & WM_TIMER & MM_TIMER \\
\hline Coding & Concision and Conveniency & More codes \\
\hline Precision & Low in general & $\begin{array}{c}\text { Maximal value is } \\
16 \mathrm{~ms} \\
\text { Minimal value is } \\
1 \mathrm{~ms}\end{array}$ \\
\hline $\begin{array}{c}\text { Sampling } \\
\text { frequency }\end{array}$ & $\begin{array}{c}\text { It is easy to appear 'Losing } \\
\text { Second' while the sampling } \\
\text { frequency is high. }\end{array}$ & $\begin{array}{c}\text { It is not easy to } \\
\text { appear 'Losing } \\
\text { Second'. }\end{array}$ \\
\hline \multicolumn{2}{|c}{}
\end{tabular}




\section{REFERENCES}

[1] LI Jing, YANG Jun-wu, QIAN Xu. Precise control of sample frequency with multimedia timer, Computer Applications, 2000, 12 (12):67-68.(in Chinese)

[2] ZHENG Cun-hong, HU Rong-qiang, ZHAO Rui-feng. Data Acquisition System Programming with Visual C++, Computer Applications and Study, 2002, 4:103-104,108. (in Chinese)

[3] YU Yong, LEI Zhi-yong. Realization of real-time measurement and control system under Windows, Modern Electronic Technology, 2005, 5(196):22-23. (in Chinese)

[4] WANG Wei, XU Guo-hua. Applications of multimedia timer in industrial control, Micro-machine and Applications, 2001, 12:8-9, 10. (in Chinese)

[5] GUAN Hong-wei, GAO Wei, CHEN Bao-xue. Solution of the real time data acquisition based on Windows environment, Applied Science and Technology, 2004, 31(4):35-37. (in Chinese) 